\title{
COMPLEMENTATION OF JORDAN TRIPLES IN VON NEUMANN ALGEBRAS
}

\author{
CHO-HO CHU AND BRUNO IOCHUM
}

(Communicated by William J. Davis)

\begin{abstract}
We show that the predual of a $J B W^{*}$-triple is complemented in the predual of a von Neumann algebra. Hence a quotientof a $J B^{*}$-triple does not contain $l_{1}$ if and only if its dual enjoys the Radon-Nikodym property. We also show that a $J B^{*}$-triple either contains $c_{0}$ or is reflexive.
\end{abstract}

\section{INTRODUCTION}

We show that every $J B W^{*}$-triple is isomorphic as a Banach space to a complemented subspace of a von Neumann algebra. Hence many Banach space properties of operator algebras do indeed pass on to Jordan triples. In fact, we prove that the predual of a $J B W^{*}$-triple $J$ is complemented in the predual of a von Neumann algebra and $J$ is even isomorphic as a Jordan triple to a 1 -complemented subtriple of a von Neumann algebra if (and only if) $J$ does not contain the exceptional Cartan factors $C^{5}$ and $C^{6}$. Moreover, if $A$ is a $J B W^{*}$-algebra not containing $C^{6}$, then it is the range of a positive contractive projection on a von Neumann algebra. This is an extension of a result of Effros and Strømer [5]. One application is that a quotient $X$ of a $J B^{*}$-triple does not contain $l_{1}$ if and only if its dual $X^{*}$ has the Radon-Nikodym property. In conjunction with this result, we also prove that a $J B^{*}$-triple either contains $c_{0}$ or is reflexive. So the Krein-Milman property and the Radon-Nikodym property are equivalent in $J B^{*}$-triples.

Briefly $J B^{*}$-triples are all those Banach spaces whose open unit balls are bounded symmetric domains [15]. These include $C^{*}$-algebras and the larger class of $J B^{*}$-algebras (Jordan $C^{*}$-algebras) as well as Hilbert spaces. A $J B^{*}$ triple is a complex Banach space $J$ equipped with a triple product $\{\cdots\}: J \times$ $J \times J \rightarrow J$ such that

(i) $\{x y z\}$ is bilinear and symmetric in $x$ and $z$, but antilinear in $y$,

(ii) $\{a b\{x y z\}\}=\{\{a b x\} y z\}-\{x\{a b y\} z\}+\{x y\{a b z\}\}$,

Received by the editors February 1, 1989.

1980 Mathematics Subject Classification (1985 Revision). Primary 17C10, 46L70, 46B22.

$K e y$ words and phrases. $J B^{*}$-triple, von Neumann algebra, complemented subspace. 
(iii) the left multiplication $\{x x\}:. J \rightarrow J$ is Hermitian and has nonnegative spectrum,

(iv) $\|\{x x x\}\|=\|x\|^{3}$.

For instance, the triple product $\{x y z\}=2^{-1}\left(x y^{*} z+z y^{*} x\right)$ in a $C^{*}$-algebra satisfies all these conditions. If, moreover, $J$ has a (necessarily unique) predual $J_{*}$, then it is called a $J B W^{*}$-triple and the $w^{*}$-topology on $J$ refers to the topology $\sigma\left(J, J_{*}\right)$. The second dual of a $J B^{*}$-triple is a $J B W^{*}$-triple. Other examples are the so-called Cartan factors $C^{k}(k=1,2, \ldots, 6)$ where $C^{4}$ is a spin factor, $C^{5}$ is the (finite-dimensional) exceptional Cartan factor consisting of $1 \times 2$ matrices over the complex Cayley numbers $\mathbf{O}$ and $C^{6}$ consists of $3 \times 3$ Hermitian matrices over $\mathbf{O}$. In fact, $C^{3}, C^{4}, C^{6}$ are $J B W^{*}$-algebras. An element $e$ in $J$ is a tripotent if $\{e e e\}=e$. It induces a Pierce decomposition $J=J_{1}(e) \oplus J_{1 / 2}(e) \oplus J_{0}(e)$ as an $l_{\infty}$-sum, where $J_{k}(e)=\{x \in J:\{e e x\}=k x\}$ $\left(k=0, \frac{1}{2}, 1\right)$ is the eigenspace of $\{e e$.$\} , called the Pierce k$-space of $e$. Also $J_{k}(e)$ is the range of the Pierce k-projection $P_{k}(e): J \rightarrow J$ where

$$
\begin{gathered}
P_{1}(e)(z)=\{e\{e z e\} e\} \\
P_{1 / 2}(e)(z)=2\left(\{e e z\}-P_{1}(e)(z)\right) \\
P_{0}(e)(z)=z-2\{e e z\}+P_{1}(e)(z) .
\end{gathered}
$$

Moreover, if $J$ is a $J B W^{*}$-triple, then the Pierce projections are $w^{*}$-continuous. A tripotent $e$ is complete if $J_{0}(e)=\{0\}$. The complete tripotents are precisely the extreme points of the closed unit ball of $J$. Tripotents abound in $J B W^{*}$-triples by the Krein-Milman theorem and they are just the partial isometries in $C^{*}$-algebras. For further properties of $J B^{*}$-triples, we refer to [10], [11], [12], [15], [18].

\section{COMPLEMENTATION IN VON NeUmanN ALGEBRAS}

Abusing the language slightly, we say that a Banach space $X$ is complemented in the space $Y$ if $X$ is isometric to a complemented subspace $Z$ of $Y$, i.e. there is a bounded projection $P: Y \rightarrow Z$. If moreover, $\|P\|=1$, we say that $X$ is 1-complemented in $Y$. We note that the predual of a Cartan factor is 1-complemented in the predual of a $J B W^{*}$-algebra. Indeed, the rectangular Cartan factor $C^{1}=L(H, K)$ consists of bounded operators between Hilbert spaces $H$ and $K$. So the predual $L(H, K)_{*}$ of $C^{1}$ is 1 -complemented in the predual of the von Neumann algebra $L(H \oplus K)$. The symplectic Cartan factor $C_{n}^{2}$ has the form $\left\{z \in L(H): z=-j z^{*} j\right\}$ where $j: H \rightarrow H$ is a conjugate linear isometric involution and $n=\operatorname{dim} H$. So its predual equals $\left\{t \in L(H)_{*}: t=-j t^{*} j\right\}$ which is 1 -complemented in $L(H)_{*}$ where $L(H)_{*}$ identifies with the trace-class operators on $H$. The Hermitian Cartan factor $C^{3}$ has the form $\left\{t \in L(H): t=j t^{*} j\right\}$ and its predual is 1-complemented in $L(H)_{*}$. Also, if $e$ is a minimal tripotent in $C^{6}$, then the Pierce projection $P_{1 / 2}(e): C^{6} \rightarrow C^{6}$ has range $C^{5}$ and so $C_{*}^{5}$ is 1 -complemented in $C_{*}^{6}$. 
Lemma 1. Let $J$ be a $J B W^{*}$-triple. Then its predual $J_{*}$ is 1-complemented in the predual of a $J B W^{*}$-algebra.

Proof. By [10], [12], $J$ admits the following $l_{\infty}$-sum

$$
J=\oplus_{\alpha} C\left(\Omega_{\alpha}, C^{\alpha}\right) \oplus J^{7} \oplus J^{8}
$$

where $C\left(\Omega_{\alpha}, C^{\alpha}\right)$ is the space of continuous functions from a hyperstonean space $\Omega_{\alpha \gamma}$ to a Cartan factor $C^{\alpha}, J^{7}$ is a continuous $J W^{*}$-algebra and $J^{8}$ is a $w^{*}$-closed right ideal of a von Neumann algebra $M$. The predual $J_{*}^{8}$ is clearly 1-complemented in $M_{*}$. The predual of $C\left(\Omega_{\alpha}, C^{\alpha}\right)$ is just the projective tensor product $L_{1}\left(\Sigma_{\alpha}\right) \hat{\otimes} C_{*}^{\alpha}$ of an $L_{1}$-space and the predual of the Cartan factor $C^{\alpha}$. Now $C_{*}^{*}$ is 1 -complemented in the predual of some $J B W^{*}$-algebras $A^{\alpha}$, it follows that $L_{1}\left(\Sigma_{\alpha}\right) \hat{\otimes} C_{*}^{\alpha}$ is 1-complemented in $L_{1}\left(\Sigma_{\alpha}\right) \hat{\otimes} A_{*}^{\alpha}$ which is the predual of the $J B W^{*}$-algebra $L_{\infty}\left(\Sigma_{\alpha}, A^{\alpha}\right)$. Thus the $l_{1}$-sum $J_{*}=\oplus_{\alpha}\left(L_{1}\left(\Sigma_{\alpha}\right) \hat{\otimes} C_{*}^{\alpha}\right) \oplus$ $J_{*}^{7} \oplus J_{*}^{8}$ is 1-complemented in the $l_{1}$-sum $\oplus_{\alpha} L_{\infty}\left(\Sigma_{\alpha}, A^{\alpha}\right)_{*} \oplus J_{*}^{7} \oplus M_{*}$.

Moreover, by [5, Lemma 2.3], every spin factor $C^{4}$ is the range of a unital positive contractive projection on the von Neumann algebra generated by $C^{4}$. By taking the bidual of the projection and noting that $C^{4}$ is reflexive, we see that $C^{4}$ embeds as a Jordan subalgebra in a von Neumann algebra $M$ and the predual of $C^{4}$ is 1-complemented in $M_{*}$. Although $C^{5}$ and $C^{6}$ are clearly complemented in von Neumann algebras by finite-dimensionality, they cannot be 1-complemented because the range of a contractive projection on a von Neumann algebra has the structure of a $J^{*}$-algebra [6; Théorème 2]. We thus reach the following conclusion.

Theorem 2. Let $J$ be a $J B W^{*}$-triple. Then its predual $J_{*}$ is complemented in the predual of a von Neumann algebra. Moreover, $J_{*}$ is 1-complemented in the predual of a von Neumann algebra if and only if $J$ does not contain $C^{5}$ and $C^{6}$.

Proof. As in Lemma 1, we write $J=\oplus_{\alpha} C\left(\Omega_{\alpha}, C^{\alpha}\right) \oplus J^{7} \oplus J^{8}$ where, by [14; 1.20], $J^{7}=\{a \in M: \Theta(a)=a\}$ where $\Theta: M \rightarrow M$ is a $w^{*}$-continuous *antiautomorphism of period 2 on a von Neumann algebra $M$. So $J^{7}$ is 1-complemented in $M$ by the bicontractive projection $2^{-1}(I d+\Theta)$. Same arguments as before complete the proof.

The Cartan factors $C^{k}$ is isomorphic as Jordan subtriple of a von Neumann algebra if and only if $k \neq 5,6$. So we have the following result by taking direct sums as before.

Corollary 3. Let $J$ be a $J B W^{*}$-triple. Then $J$ is isomorphic as a Banach space to a complemented subspace of a von Neumann algebra. Moreover, $J$ is isomorphic as a Jordan triple to a 1-complemented subtriple of a von Neumann algebra if and only if it does not contain $C^{5}$ and $C^{6}$.

Remark 4 . In the case of a $J B W^{*}$-algebra $A$ without the summand $C\left(\Omega, C^{6}\right)$, it can be embedded as a Jordan subalgebra of a von Neumann algebra $M$ and is 
1-complemented by a $w^{*}$-continuous positive contractive projection on $M$. Indeed, we can write $A=\oplus_{\alpha} C\left(\Omega_{\alpha}, C^{\alpha}\right) \oplus B$ where $C^{\alpha}$ are spin factors and $B$ is a $J W^{*}$-algebra 1-complemented in a von Neumann algebra by a $w^{*}$-continuous bicontractive projection $E[9 ; 5.3 .5,6.3 .14,7.3 .5]$. As remarked before, $C^{\alpha}$ is the range of a $w^{*}$-continuous contractive projection $P_{\alpha}: M \rightarrow M$ on a von Neumann algebra $M$ whose predual $Q_{\alpha}$ has $C_{*}^{\alpha}$ for range. Now $P_{\alpha}$ lifts to a projection

$$
\begin{gathered}
E_{\alpha}: C\left(\Omega_{\alpha}, M^{\alpha}\right) \rightarrow C\left(\Omega_{\alpha}, C^{\alpha}\right) \text { with predual } \\
Q_{\alpha} \otimes 1: L_{1}\left(\Sigma_{\alpha}\right) \hat{\otimes} M_{*}^{\alpha} \rightarrow L_{1}\left(\Sigma_{\alpha}\right) \hat{\otimes} C_{*}^{\alpha} .
\end{gathered}
$$

Therefore $A$ is the range of the $l_{\infty}$-sum of the projections $E_{\alpha}$ and $E$ which is $w^{*}$-contiunuous and contractive, hence positive by $[9 ; 4.4 .13]$.

\section{BANACH SPACE PROPERTIES}

It is well known that a Banach space does not contain $l_{1}$ if and only if its dual has the weak Radon-Nikodym property $[14,16]$. Applying Theorem 2, we have the following sharper result for $J B^{*}$-triples which is an improvement of [2; Theorem 2], see also [17].

Theorem 5. Let $X$ be a quotient of a $J B^{*}$-triple $J$. Then $X$ does not contain $l_{1}$ if and only if $X^{*}$ has the Radon-Nikodym property.

Proof. First $X^{*}$ is isometric to a subspace of $J^{*}$ and by Theorem 2, $J^{*}$ is complemented in the predual $M_{*}$ of a von Neumann algebra $M$. But $M_{*}$ is complemented in $\left(M_{*}\right)^{* *}$ and every separable subspace of $M_{*}$ is contained in a separable complemented subspace of $M_{*}$ (see [7; Appendix]), therefore the closed unit ball of $X^{*}$ has the Radon-Nikodym property if and only if it is strongly regular in the sense of [7] which in turn is equivalent to $X \not \supset l_{1}$ by [7; VIII.4 and VIII.18].

Finally we prove that every $J B^{*}$-triple either contains $c_{0}$ or is reflexive.

Theorem 6. Let $J$ be a $J B^{*}$-triple. Then the following conditions are equivalent:

(i) $J$ has the Krein-Milman property,

(ii) $J$ does not contain $c_{0}$,

(iii) $J$ is reflexive,

(iv) $J$ has the Radon-Nikodym property.

Proof. (ii) $\Rightarrow$ (iii). Take any $x$ in $J$. Then the $J B^{*}$-triple $J_{x}$ generated by $x$ is an Abelian $C^{*}$-algebra [15]. Now $J_{x} \not \supset c_{0}$ implies $J_{x}$ is finite dimensional since the identity map on $J_{x}$ is weakly compact [1] and so $J_{x}$ contains mutually orthogonal tripotents. Let $\left\{e_{1}, \ldots, e_{n}\right\}$ be a maximal family of orthogonal tripotents in $J$. As in [3; p. 186], we have $n<\infty$ for otherwise $J$ would contain a copy of $c_{0}$. Let $e=e_{1}+\cdots+e_{n}$. We show that $e$ is a complete tripotent in $J$. Indeed, if $z \in J_{0}(e)$ and $z \neq 0$, then by considering $J_{z} \subset$ $J_{0}(e)$, there is a nontrivial tripotent $u \in J_{z} \subset J_{0}(e)$. By orthogonality, we 
have $e_{k} \in J_{1}(e)$ which implies $J_{0}(e) \subset J_{0}\left(e_{k}\right)$ (cf. [11; 1.14]) and hence $u \in J_{0}\left(e_{k}\right)$, that is, $u$ is orthogonal to $e_{k}$ for $k=1, \ldots, n$. This contradicts maximality. So $J_{0}(e)=\{0\}$. Recall that the Pierce $k$-space $J_{k}^{* *}(e)$ in $J^{* *}$ is the range of the Pierce projection $P_{k}(e): J^{* *} \rightarrow J^{* *}$ and as $e \in J$, we also have $J_{k}(e)=P_{k}(e) J$. But $P_{k}(e)$ is $w^{*}$-continuous, it follows that $J_{k}(e)$ is $w^{*}$-dense in $J_{k}^{* *}(e)$. In particular, $J_{0}^{* *}(e)=\{0\}$ and $e$ is a complete tripotent in $J^{* *}$. Now $J_{1}(e) \not \supset c_{0}$ implies that $J_{1}(e)$ is relfexive by [4; Theorem 8]. The predual of $J_{1}^{* *}(e)$ is $J^{*} / J_{1}^{* *}(e)^{0}$ where $J_{1}^{* *}(e)^{0}$ is the polar in $J^{*}$ [11; 3.7]. As $J_{1}(e)$ is $w^{*}$-dense in $J_{1}^{* *}(e), J_{1}^{* *}(e)^{0}$ coincides with the polar $J_{1}(e)^{0}$ of $J_{1}(e)$ in $J^{*}$. Thus $J_{1}(e)^{*}=J^{*} / J_{1}(e)^{0}$ is the predual of $J_{1}^{* *}(e)$ and hence $J_{1}(e)=J_{1}(e)^{* *}=J_{1}^{* *}(e)$. In other words, $J_{1}(e)$ is the Pierce 1-space of $e$ in $J^{* *}$.

Now $J_{1}(e)$ is a finite $l_{\infty}$-sum $A_{1} \oplus \cdots \oplus A_{n}$ of relfexive Cartan factors [4; p. 457]. Hence, by $[10 ; 1.12]$, we have an $l_{\infty}$-sum $J^{* *}=U_{1} \oplus \cdots \oplus U_{n}$ of $w^{*}$-closed triple ideals with $A_{j}=U_{j} \cap J_{1}(e)$. If we write $e=u_{1}+\cdots+u_{n}$, then $u_{j}$ is a complete tirpotent in $U_{j}$ such that its Pierce 1-space $\left(U_{j}\right)_{1}\left(u_{j}\right)$ is just $A_{j}$. To complete the proof, we show that $J^{* *}$ is reflexive. It suffices to show that each $U_{j}$ is reflexive. First $U_{j}$ is a Cartan factor by $[11 ; 4.6]$. Note that the Cartan factors $C^{4}, C^{5}$, and $C^{6}$ are reflexive. If $U_{j}$ is a rectangular Cartan factor, then by $[10 ; 5.5], A_{j}=\left(U_{j}\right)_{1}\left(u_{j}\right)$ contains a complete rectangular grid $\left(e_{l k}\right) \quad(l, k \in I)$ with $u=\sum_{l \in I} e_{l l}$. Also $U_{j}=L(H, K)$ with $\operatorname{dim} K$ equals to card $I$. As $A_{j} \not \supset c_{0}, A_{j}$ cannot contain infinitely many mutually orthogonal tripotents and hence card $I<\infty$. So $U_{j}$ is reflexive in this case. If $U_{j}$ is symplectic, then by $[10 ; 6.1] A_{j}=C_{2 n}^{2}$ with $n<\infty$ and $U_{j}$ is either $C_{2 n}^{2}$ or $C_{2 n+1}^{2}$, and hence it is reflexive. Finally, if $U_{j}$ is hermitian type, i.e. of type $C^{3}$, then by $[10 ; 7.1], u_{j}$ is unitary which means $U_{j}=A_{j}$ and so $U_{j}$ is reflexive.

\section{ACKNOWLEDGMENTS}

This work was done while the first author was visiting Centre de Physique Théorique, C.N.R.S. in Marseille. He acknowledges a research grant from the European Science Exchange Programme of the Royal Society and C.N.R.S. as well as financial support from Université de Provence and he thanks warmly colleagues in Marseille for their hospitality. We also thank Dr. G. Godefroy for useful conversations.

\section{REFERENCES}

1. C. A. Akemann, P. G. Doods and J. L. B. Gamlen, Weak compactness in the dual space of a $C^{*}$-algebra, J. Funct. Anal. 10 (1972), 446-450.

2. T. Barton and G. Godefroy, Remarks on the predual of a $J B^{*}$-triple, J. London Math. Soc. 34 (1986), 300-304. 
3. T. Barton and R. M. Timoney, Weak*-continuity of Jordan triple products and applications, Math. Scand. 59 (1986), 177-191.

4. C. H. Chu and B. Iochum, Weakly compact operators on Jordan triples, Math. Ann. 281 (1988), 451-458.

5. E. G. Effors and E. Størmer, Positive projections and Jordan structure in operator algebras, Math. Scand. 45 (1979), 127-138.

6. Y. Friedman and B. Russo, Algèbres d'opérateurs sans ordre: solution du problème du projecteur contractif, C.R. Acad. Sci. Paris 296 (1983), 393-396.

7. N. Ghoussoub, G. Godefroy, B. Maurey and W. Schachermayer, Some topological and geometrical structures in Banach spaces, Mem. Amer. Math. Soc. No. 378 (1987).

8. N. Ghoussoub and E. Saab, On the weak Radon-Nikodym property, Proc. Amer. Math. Soc. 81 (1981), 81-84.

9. H. Hanche-Olsen and E. Størmer, Jordan operator algebras, Pitman, Marshfield, MA, 1984.

10. G. Horn, Classification of $J B W^{*}$-triples of type I, Math. Z. 196 (1987), 271-291.

11. Characterization of the predual and ideal structure of a $J B W^{*}$-triple, Math. Scand. 61 (1987), 117-133.

12. G. Horn, and E. Neher, Classification of continuous $J B W^{*}$-triples, Trans. Amer. Math. Soc. 306 (1988), 553-578.

13. B. Iochum, Cônes autopolaires et algèbres de Jordan, Lecture Notes in Math. vol. 1049, Springer-Verlag, 1984.

14. L. Janicka, Some measure-theoretical characterization of Banach spaces not containing $l_{1}$, Bull. Acad. Polonaise Sci. 27 (1979), 561-565.

15. W. Kaup, A Riemann mapping theorem for bounded symmetric domains in complex Banach spaces, Math. Z. 183 (1983), 503-529.

16. K. Musial and C. Ryll-Nardzewski, Liftings of vector measures and their applications to RNP and WRNP, Lecture Notes in Math. vol. 645, Springer-Verlag, 1977, pp. 162-171.

17. C. Niculescu, A note on weakly compact operators in $C^{*}$-algebras, Rev. Roumaine Math. Pures Appl. 25 (1980), 631-634.

18. H. Upmeier, Symmetric Banach manifolds and Jordan $C^{*}$-algebras, North-Holland Math. Studies, Vol. 104, North-Holland, Amsterdam, 1985.

Goldsmiths' College, London SE14 6NW, England

Université de Provence et CNRS-CPT luminy, Case 907, F-1 3288 Marseille, France 